\title{
Vers une sociologie économique relationnelle
}

L'Empire de la valeur d'André Orléan (Seuil, 2011)

\section{Philippe Steiner}

\section{(2) OpenEdition}

\section{Journals}

Édition électronique

URL : http://journals.openedition.org/ress/2307

DOI : $10.4000 /$ ress. 2307

ISSN : 1663-4446

Éditeur

Librairie Droz

\section{Édition imprimée}

Date de publication : 1 décembre 2012

Pagination : 207-218

ISBN : 978-2-600-01704-6

ISSN : 0048-8046

\section{Référence électronique}

Philippe Steiner, «Vers une sociologie économique relationnelle », Revue européenne des sciences sociales [En ligne], 50-2 | 2012, mis en ligne le 01 janvier 2016, consulté le 30 avril 2019. URL : http:// journals.openedition.org/ress/2307 ; DOI : 10.4000/ress.2307 
Le dossier qui suit offre un regard croisé et interdisciplinaire sur l'ouvrage d’André Orléan à travers trois contributions critiques émanant d'un sociologue, Philippe Steiner, d'un économiste, Richard Arena, et d'un philosophe, Marc Hunyadi. Il est l'occasion d'un éclairage nouveau et d'une discussion critique et argumentée à l'endroit de quelquesunes des thèses refondatrices de L'Empire de la valeur.

- Philippe Steiner entreprend de montrer que l'analyse des échanges marchands à laquelle se livre André Orléan n'est pas sans faire écho aux développements contemporains de la sociologie relationnelle et qu'elle pose, ce faisant, les jalons d'une véritable sociologie économique relationelle.

- Richard Arena entend, de son côté, mettre les thèses d'André Orléan à l'épreuve d'une analyse en terme de coût et de répartition des revenus. Il vise ainsi à intégrer la dimension productive et technologique du système de production capitaliste dans le projet de redéfinition de la valeur, par delà la seule composante marchande de l'échange.

- Marc Hunyadi, enfin, soulève une ambiguïté fondamentale dans l'analyse marxiste de la valeur-travail et vise à contester la thèse d’André Orléan selon laquelle Marx aurait fait à son tour du travail, dans le cours même de sa critique du fétichisme de la valeur, une substance.

\section{VERS UNE SOCIOLOGIE ÉCONOMIQUE RELATIONNELLE L'Empire de la valeur d'André Orléan (Seuil, 20II)}

par Philippe Steiner

Université Paris-Sorbonne/ Gemass \& Institut universitaire de France ph.steiner@free.fr

Contributeur de longue date du courant de l'économie des conventions, éditeur d'un volume synthétique ${ }^{1}$ sur le sujet dans lequel Mark Granovetter, un sociologue emblématique s'il en est de la sociologie économique contemporaine, faisait parti des contributeurs, membre actif du comité de pilotage du Groupement de recherche «Économie et sociologie» du CNRS ${ }^{2}$, président de l'Association Française d'Économie Politique $(\mathrm{AFEP})^{3}$, André Orléan est un économiste bien connu du sociologue intéressé par l'éco-

I André Orléan (éd.), 1992, Analyse économique des conventions, Paris, PUF ; réédition dans la collection Quadrige, Paris, PUF, 2002.

2 Fondé en 2004, le GDR «Économie et Sociologie» est actuellement dirigé par Alexandra Bidet (Centre Maurice Halbwachs, Paris) et Florence Jany-Catrice (Centre Lillois d'Études et de Recherches en Socio-Économie, Lille): son objectif est de promouvoir les échanges entre les chercheurs des deux disciplines.

3 Voir la présentation de cette association sur le site: http://www.assoeconomiepolitique.org 
nomie. Ses travaux sur la finance figurent parmi ceux qui permettent aux sociologues de discuter fructueusement avec les économistes lorsqu'il s'agit de la sociologie des marchés. Nourri de références aux œuvres sociologiques (tout particulièrement, Émile Durkheim, Georg Simmel et François Simiand) L'Empire de la valeur. Refonder l'économie mérite de retenir l'attention du sociologue. Tout en suivant le fil menant de la critique de l'économie politique (I), à la mise en avant de la monnaie et des relations qui l'instituent (2), que la notion de «liquidité» actualise sur les marchés financiers contemporains (3), je me propose de mettre l'accent, unilatéralement, sur un thème transversal de l'ouvrage de manière à montrer qu'Orléan ouvre des perspectives stimulantes pour une sociologie économique relationnelle.

\section{Critique de l'économie}

En s’attaquant à la valeur, Orléan porte le fer au cœur de la théorie économique, sous sa forme classique (Karl Marx) ou néoclassique (Léon Walras), en ciblant la théorie du marché. Celui-ci est une forme de socialisation basée sur la séparation : les acteurs du marché sont indépendants les uns des autres, produisent, échangent, etc., sans aucune planification ou coordination ex ante. Comment dans ces conditions assurer la compatibilité des décisions et donner une cohérence globale à ces dernières? L’idée forte avancée par Orléan est quavec le travail abstrait comme avec l'utilité, la théorie de la valeur répond à cette question en se référant à un phénomène situé en dehors du social et de l'historique - ce que les philosophes appellent une «substance» (EV, p. 24-27). De là une conséquence remarquable de la théorie de la valeur: la prise en compte des échanges effectifs n'est plus nécessaire pour expliquer la formation du système de prix (EV, p. 27). Dans le cas de Marx, les données issues de à la production - les quantités de travail social abstrait - suffisent à fixer les valeurs. La cohésion marchande repose alors sur la division du travail (EV, p. 37), puisque cette dernière représente l'ensemble des travaux concourant à définir les quantités de travail contenues dans les marchandises. La remarque s'applique à l'œuvre de Durkheim qui étudie lui aussi la cohésion marchande par l'intermédiaire de la division du travail et ne dit rien des relations d'échange. Même lorsqu'il étudie le contrat et ses dimensions non contractuelles, il n'examine jamais comment se déroulent les échanges que les contrats mettent en forme juridique. Et pour la sociologie économique durkheimienne, cela signifie que Durkheim n’a jamais pu se saisir du marché comme un espace social spécifique, avec ses formes de contraintes, constitutives du social dans le domaine de l'économique, au-delà de ce qui, à défaut, apparaît comme la recherche des plaisirs sans règles de l'eudémonismet. Dans le cas de Walras, ce sont les préférences des individus, préférences considérées comme exogènes, qui permettent de contourner les échanges, et, du même coup, la monnaie. Orléan renouvelle ainsi la

4 II n'en va pas de même pour François Simiand et Maurice Halbwachs, qui se sont intéressés aux échanges concrets, respectivement sur le marché du travail (formation des salaires) et sur le marché des biens de consommation (alimentation, vêtement, logement). 
critique de l'homo œconomicus en expliquant qu’avant même d’être le héros conséquentialiste, infatigable et surinformé, de la maximisation sous contrainte, l'acteur économique est caractérisé par le fait de n'avoir pas d’autres affects que ceux que suscitent en lui les biens rares: «la satisfaction que procurent les biens consommés est sa passion exclusive, son unique intérêt, son seul affect» (EV, p. 59). La coordination passe donc par les objets et c'est l'accord sur les prix formés par tâtonnement grâce à une instance centralisatrice - le commissaire priseur - et sans que les acteurs économiques n’interagissent directement les uns avec les autres qui permet d'y parvenir ${ }^{5}$.

En quoi tout ceci peut-il intéresser le sociologueau-delà de ce qu'il peut apprendre sur les théories économiques? Au fait que cette critique de la théorie de la valeur est une invitation à prendre au sérieux les relations sociales qui font le marché, puisqu'une fois enlevés le rapport aux objets et la division du travail, il ne reste pas d'autre point d'appui pour comprendre les échanges marchands. Présente tout au long de l'ouvrage (EV, p. I4, 22, 4I, 52, 228, etc.), cette thèse indique qu'Orléan entend rompre avec la primauté accordée aux grandeurs (la valeur, les prix) pour donner aux relations marchandes toute leur place. Il s'agit d'en finir avec la sous-estimation des échanges (EV, p. 32-36) et de mettre les relations sociales au centre de l'analyse. Un tel changement de perspective entraîne une modification en profondeur de l'approche économique car avec les relations sociales les opinions et les rapports de force (EV, p. 239) deviennent des éléments indispensables pour rendre compte de ce qu'il se passe sur le marché.

Cette formulation du problème est de grande portée une fois que, à l'instar de Durkheim, Orléan donne aux relations sociales le statut de fait sui generis car elles produisent des résultats spécifiques que la théorie économique a exclus de son approche : en prenant les relations entre les acteurs du marché comme point de départ, l'acteur économique n'est plus indépendant, et ne forme plus ses préférences dans un splendide isolement. Les préférences ne peuvent plus jouer le rôle de condensé de social servant de point d'appui aux constructions économiques fondées sur le primat et l'autonomie des décisions individuelles. En lieu et place, écrit Orléan, «les interactions marchandes ont de l’importance; elles façonnent les individus et leurs croyances. La valeur ne préexiste pas au marché» (EV, p. 259).

L'acteur et ses préférences résultent des relations marchandes, et rendent inopérante la stratégie d'isolement de la théorie économique vis-à-vis des autres sciences sociales. Comme l'a écrit il y a un siècle Durkheim lorsqu'il ferraillait avec les économistes de son temps: «Qu’on le veuille ou non, qu'elles soient un bien ou un mal, les sociétés existent. C'est au sein de sociétésconstituées que se manifeste la vie économique. La logique ne peut rien contre un fait qui complique, il est vrai, les données du problème, mais dont il n'est pas possible de faire abstraction» (Durkheim, I886, p. 208). Mais, demandera- 
t-on, quelles sont les traductions concrètes de cette mise en avant des relations sociales marchandes? Comment la complexité accrue par une approche soucieuse des structures relationnelles peut-elle être traitée par une théorie économique réintégrant le giron des sciences sociales ? Orléan n'est pas en peine de répondre à cette attente : avec les relations émerge une classe spécifique de marchés qu'il appelle les «marchés à rétroactions positives » (EV, p. 26I), c'est-à-dire les marchés dans lesquels les règles du «marché standard» des économistes ne fonctionnent plus, notamment les forces de rappel qui font qu'une hausse du prix entraîne une baisse de la demande du bien au profit des biens substituables. Cette classe de marchés est vaste puisqu'elle comporte les marchés avec asymétrie d’informations (étudiés par George Akerlof), les marchés avec rendement croissant (étudiés par Brian Arthur), les marchés où s'échangent des biens de prestige (bien connus depuis les travaux de Thorstein Veblen), les marchés financiers chargés de donner réalité à la notion de liquidité (étudiés par John Maynard Keynes, et approfondis par Orléan).

La liste est donc vaste des marchés dans lesquels la prise en compte des relations marchandes est nécessaire pour expliquer leur fonctionnement. Orléan ne rejette donc pas en bloc toute la théorie économique, ni sa propension à modéliser, mais il en conteste la généralité (EV, p. I3 et I5) dont l'échec de la théorie de la stabilité est la manifestation (EV, p. I47). Il est des régimes du marché qui sont redevables de la théorie économique, lorsque les objets ont des qualités bien définies, que le futur est maîtrisable par des lois de probabilités (EV, p. 23I et 239), que l'institution monétaire ne fait pas difficulté. Les modèles de la théorie monétaire de Don Patinkin sont recevables lorsque la monnaie fait l'objet d'une «confiance méthodique» produite par le cours routinier des transactions (EV, p. I72) et que la monnaie rend le service de la liquidité aux échangistes (EV, p. I03).

Si la critique est soucieuse des apports de la théorie économique, elle n’en est pas moins forte en cantonnant ces derniers au cas limite dans lequel l'étroitesse des hypothèses institutionnelles de l'économie est recevable: alors l'objectivité marchande fonctionne à plein avec un acteur rationnel qui sait maximiser et, surtout, ce qu'il maximise, des préférences stables, des biens dont les qualités sont connues, un futur maîtrisé par les probabilités et des interactions entre les acteurs ne produisant rien de substantiellement neuf (EV, p. 260). Au-delà de ce cas limite, les relations sociales, et donc les opinions et les rapports de domination, comptent et d’autres cadres institutionnels doivent être construits.

\section{La monnaie et la valeur}

La monnaie occupe une place de choix dans la stratégie de refondation d’Orléan: «Notre projet de refondation trouve ici sa définition: saisir la valeur marchande dans son autonomie, sans chercher à l'identifier à une grandeur préexistante, comme l'utilité, le travail ou la rareté. Cette autonomie qui donne à voir la valeur en majesté, dans la plénitude de sa puissance, c'est grâce à la monnaie qu'elle l'obtient» (EV, p. I2-I3). La monnaie fait l'objet, elle aussi, d'une approche relation- 
nelle. Cela apparaît aussi bien dans le fonctionnement des échanges que permet cette institution essentielle de l'économie de marché, que dans le processus conduisant à son apparition. La monnaie est une relation dont la trace est donnée dans les comptes des agents: décaissement pour celui qui a acheté le bien a au prix $\mathrm{p}_{\mathrm{a}}$, encaissement pour celui qui l'a vendu (EV, p. I53). À ce premier niveau relationnel, s'en rajoute un deuxième reposant sur le fait que la monnaie est une «puissance d'achat», un droit reconnu par la société de puiser dans le flux des richesses produites car le détenteur de monnaie détient le bien sur lequel converge les désirs des membres du collectif monétaire (EV, p. I90 et I93). La dimension relationnelle met alors en jeu la confiance et la promesse - faits relationnels par excellence - confiance dans le fait que, dans le futur, même très proche, la promesse sera bien reçue par les membres du collectif comme un droit, quantitativement défini, de puiser dans le flux des biens et services, ce qui définit le concept, central chez Orléan, de liquidité : «la liquidité n’est pas une substance mais un mode de relation à autrui, un lien social par lequel est reconnue entre les protagonistes l'existence d'une communauté d'intérêts mais transfiguré sous la forme d'un désir d’objets » (EV, p. 158). Elle est de ce fait un pouvoir sur autrui (EV, p. 189).

La monnaie support de la liquidité est également le résultat d'une structure relationnelle très particulière qu'Orléan éclaire par une étude de la «genèse conceptuelle» de la monnaie. Il faut que les relations entre les individus sur le marché se polarisent sur un même objet auquel, en conséquence de la polarisation, chacun accorde la caractéristique de liquidité, c'est-à-dire d'être recherché par lui parce que unanimement recherché par autrui. C'est l'exemple même de la logique autoréférentielle: «Est liquide pour un individu ce que les autres considèrent comme liquide et désirent comme tel» (EV, p. I55). On retrouve donc ici l'hypothèse mimétique bien connue des lecteurs des travaux antérieurs d'Orléan et de Michel Aglietta (2002). Cette hypothèse est ici étayée sur d’autres travaux que ceux mobilisés précédemment et, notamment, sur les passages célèbres des Formes élémentaires de la vie religieuse dans lesquels Durkheim explique l'apparition du sacré par l'intermédiaire des phénomènes d’effervescence collective, moments dans lesquels les individus ressentent le pouvoir du collectif comme un élément qui les domine, les entraîne, les fortifie. Cette référence à Durkheim n'est pas un simple changement de forme dans l'argumentation, puisque Orléan y trouve le moyen de montrer que la valeur économique portée par l'institution monétaire est une des formes prises par la valeur en tant que concept général désignant ces produits collectifs qui exercent une domination sur les membres du collectif qui lui ont donné naissance (EV, p. 199). Cela constitue une belle démonstration de l'idée selon laquelle l'économie politique est une science sociale et qu'il est possible de penser d'une même manière les valeurs ayant cours dans différents domaines de la vie sociale. Dans le cas du sacré (Durkheim) comme dans celui de la monnaie (Orléan), on trouve la même situation relationnelle que l'on peut exprimer en termes de rapports : collectif / individu $\approx$ sacré / profane $\approx$ monnaie / marchandises. La monnaie est la forme du collectif dans le domaine des relations marchandes de même 
que le sacré est celle dans le domaine du religieux. Et ce sont des systèmes de relations spécifiques - les effervescences collectives dans le cas de Durkheim, les comportements mimétiques de la «puissance de la multitude » dans le cas d’Orléan - qui produisent par autoréférentialité cette puissance du collectif.

De même que Durkheim avait insisté sur le fait que tout n'est pas contractuel dans le contrat et donc qu'il existe des éléments extérieurs aux obligations mutuelles sur lesquelles les échangistes conviennent, Orléan souligne que la monnaie ne résulte pas d'un contrat. Dans l'un et l'autre cas, des éléments d'origines collectives interviennent pour donner une force particulière aux relations entre les échangistes. En ce sens «on ne saurait identifier le lien marchand à un lien contractuel» (EV, p. 190). La deuxième conséquence importante tient au fait qu'en plaçant la coordination par la monnaie - l'accord sur un même objet définissant la liquidité - avant la coordination par les prix, Orléan place l'intérêt, passion modérée et gouvernable, selon la démonstration classique d'Albert Hirschman (1977), face à son contraire, le désir infini de puissance que procure la monnaie. Un bref développement peut ici servir à montrer la fécondité de cette manière de faire.

Le marché walrassien, dit Orléan, a la remarquable propriété de produire de la modération, les acteurs du marché ne s'intéressant qu'aux objets et à la satisfaction qu'apporte leur consommation (EV, p. 59). La modération repose sur deux phénomènes. Le premier est de l'ordre de la relation à soi-même, d'un autocontrôle engendré par la pratique marchande : l'acteur rationnel non seulement ne se laisse influencer par personne, mais il est également sous l'emprise de son objectif de maximisation de l'utilité. Orléan a parfaitement raison de souligner le fait «qu’en imposant que jamais le désir des acteurs ne s'écarte de ce que dicte le calcul de l'utilité, cette hypothèse [de recherche d'un maximum d'utilité] a pour effet d'introduire un puissant facteur de modération dans la lutte concurrentielle: ce faisant la violence acquisitive se trouve de facto étroitement encadrée» (EV, p. 58). L’autocontrainte portée par la recherche optimale de l'utilité n'est pas un phénomène que Walras ignorait puisqu'elle a été explicitement élaborée comme telle par Herman Gossen dans ses travaux d'économie mathématique au cours desquels il a mis au jour les lois - désormais connues comme les «lois de Gossen »- que doivent suivre les individus cherchant à tirer le plus d'utilité possible des biens à leur disposition. Ce n'est donc pas par hasard, et pas uniquement pour sa dimension mathématique, que Walras a pu être intéressé par cet ouvrage au point de le traduire en français ${ }^{6}$ : il y trouvait aussi l'affirmation de cette modération que recelait son approche conduite en termes d'équilibre général. Mais la modération a aussi une origine collective issue du système de relations marchandes. En effet, le marché est un lieu social dans lesquels les relations marchandes contraignent les individus en mettant leurs désirs face aux désirs d'autrui, en faisant que

6 Le livre de Gossen (1854) est traduit par Walras en collaboration avec Charles Secrétan. Cette traduction n'a pas paru à l'époque, mais plus d'un siècle plus tard, en annexe des Fuvres complètes de Léon Walras. 
face à celui qui veut acheter le moins cher possible, prend place le vendeur qui veut vendre le plus cher possible. Cette situation impose une modération dans l'expression des appétits de richesse qu'enregistre la maximisation sous contrainte de leur utilité.

Ce régime de modération a un double: la volonté de puissance marchande portée par la détention la plus large possible, proprement sans limite, de la monnaie, l'objet sur lequel converge les désirs unanimes des échangistes, objet permettant d'exercer une domination sur autrui puisque permettant de puiser aussi largement que possible dans le flux des biens et services, et d'afficher son prestige sous forme de sa place dans une hiérarchie mondiale des plus grosses fortunes. Atteindre la première place ne suffit pas même à éteindre la recherche de la monnaie, car l'écart avec le second n'est jamais assez grand pour cela. La comparaison envieuse veblennienne est, elle aussi, de nature relationnelle.

\section{Marchés financiers}

Le marché financier sur lesquels s'échangent les actifs qui sont des droits sur les revenus futurs, engage le rapport au temps. Il fait lui aussi l'objet d'une approche conduite en termes relationnels dans la mesure où il est un exemple des marchés à rétroactions positives - les bulles sont caractérisées par le fait qu'il est rationnel d'acheter aujourd'hui un actif dont on pense qu'il est surévalué si on pense aussi que d'autres seront près à l'acheter encore plus cher demain.

Le marché financier secondaire où s'échangent les actifs financiers a pour fonction de rendre liquide ce qui ne l'est pas. Le capital investi n'est pas liquide, par contre les actifs qui sont des droits sur les revenus engendrés par ces capitaux peuvent eux changer de main et acquérir cette propriété de liquidité. (EV, p. 273-274). Orléan s'appuie sur le chapitre 12 de la Théorie générale de Keynes reprenant les deux types de comportement qui peuvent se concevoir sur ce marché : le point de vue de l'entrepreneur où le profit recherché dépend du flux de revenus futurs rapporté au capital investi et le point de vue du spéculateur cherchant un profit à partir des variations de prix des actifs financiers (EV, p. 266-268)7. Orléan défend vigoureusement l'idée selon laquelle le comportement de spéculation ne dépend pas de la valeur fondamentale et que les marchés financiers n’ont pas la propriété d’efficience que la théorie financière moderne leur attribue. Les marchés financiers ne sont pas le lieu de la formation d’un prix qui fait se rencontrer le point de vue de l'entrepreneur et celui du spéculateur, mais plutôt celui de la formation d'une

7 Le présent ouvrage ne reprend pas certains des développements économico-politiques que, dans un précédent ouvrage, Orléan (1999, chap. 4) associait à ces deux approches: le marché financier met aussi en tension l'individualisme patrimonial, celui fondé sur la rémunération de ses actifs, et la citoyenneté liée à la monnaie, à l'État et à une localisation définie donnant lieu à paiement de l'impôt. Certes, ces développements sont extérieurs à l'objet du présent ouvrage, mais, après la crise financière issue de la bulle formée sur le marché immobilier américain, ces développements gardent toute leur valeur et méritent d'être signalés. 
convention d'évaluation des actifs grâce à un processus relationnel spécifique que Keynes avait présenté sous le nom du «concours de beauté». Il ne sagit pas de savoir définir la valeur fondamentale mieux que ne le font les autres intervenants du marché, mais d'être capable de conjecturer la valeur sur laquelle le collectif que forment ces intervenants va se fixer. Les relations entre les échangistes ne sont pas tant des relations dans lesquelles les préférences de i dépendent des préférences de j, qui elles mêmes dépendent des préférences de k, etc., mais plutôt d'un jeu relationnel dans lesquels les préférences de i, j, k, etc., dépendent de ce qu'ils pensent que préfère le collectif qu'ils forment ${ }^{8}$. Dans les termes du «concours de beauté » de Keynes il ne s'agit pas de dire quel visage est le plus beau de son point de vue, ni de trouver le visage que l'acteur i, j, etc. trouve le plus beau, mais de conjecturer ce que le collectif des réponses définit comme le plus beau visage.

La concurrence porte alors directement sur la puissance marchande, sur la liquidité, c'est-à-dire sur les relations sociales qui font que tel objet est doté de cette propriété de fixation des désirs du collectif. Aussi, il n’est pas surprenant que les profits de la spéculation soient des profits faits sur les relations marchandes elles-mêmes, lesquelles sont le lieu par excellence de l'échappée de la modération marchande pour laisser libre cours aux gains exorbitants du monde de la finance qui représente à lui seul plus de $26 \%$ des membres du premier millime de la distribution des revenus en France alors qu'ils ne pèsent que $3 \%$ de la population active (Godechot, 201 I).

\section{Conclusion}

En mettant l'accent sur la dimension relationnelle des échanges marchands et de la monnaie, Orléan jette un pont solide avec les développements contemporains de la sociologie relationnelle qui tâche de se débarrasser des «substances» (individus, objets, positions sociales) en tant que catégories préalables de l'étude du social: la sociologie relationnelle de White suggère de définir les instances sociales par l'intermédiaire de la définition de positions (les blockmodels) dans un réseau relationnel; la sociologie de Bourdieu définit les positions sociales par l'ensemble des oppositions (les différents capitaux, leurs volumes) structurant un champ social; la sociologie de Latour vise à tracer les réseaux d'actants (humains ou non humains) faisant faire des choses inattendues à d'autres actants. La refondation que propose Orléan entre de plain-pied avec ces développements de la sociologie relationnelle contemporaine dont on sait par ailleurs que la dimension de sociologie économique y est fortement développée. 
Le rapprochement ne signifie pourtant pas une confusion entre les deux disciplines. Le sociologue de l'économie peut émettre des réserves quant à l'importance accordée à l'hypothèse du mimétisme comme forme relationnelle susceptible de donner aux systèmes d'échanges une structure relationnelle qui lui fait défaut, notamment dans les périodes de crise. Cette dimension de la refondation proposée par Orléan est un peu unilatérale et, surtout, un peu trop abstraite pour donner prise à des travaux empiriques de la part des sociologues économistes, qu'ils soient intéressés par les «acteurs du marché » et les systèmes relationnels découlant de tout l'appareil gestionnaire sans lequel les marchés contemporains n'existeraient tout simplement pas (Cochoy \& Dubuisson-Quellier, 2000), ou qu'ils mettent l'accent à la suite du travail fondateur de Lucien Karpik (2007) sur une vaste gamme de dispositifs de jugement grâce auxquels les consommateurs font face à l'incertitude marchande lorsqu'il s'agit pour eux de trouver le «bon » produit. On peut également penser que le haut niveau d’abstraction de la réflexion d'Orléan demande un important travail empirique afin de rencontrer les travaux sociologiques sur tel ou tel point de la relationalité marchande. On peut en trouver d'ores et déjà des exemples qui permettent d'être optimistes sur ce point, comme c'est le cas des recherches rassemblées sur la sociologie des valeurs (Becker \& Aspers, 20I I), ou sur les dispositifs permettant de construire la modération de l'action intéressée par mise à l'écart des addictions ou de la détresse (Steiner \& Trespeuch, 2013).

La critique de la théorie classique et néoclassique de la valeur est portée dans cet ouvrage à un stade qui ne sera pas dépassé de si tôt. Le compliment vaut aussi limite puisque les développements récents de la théorie économique - économie expérimentale, l’ingénierie économique des market designers ou, encore, l'économétrie adossée à de grosses bases de données empiriques - ne sont pas directement pris en compte par Orléan, alors que ces derniers occupent une place grandissante depuis que les économistes ont pris acte de l'effondrement des grandes structures théoriques, la théorie de la valeur travail de Marx comme la théorie de l'équilibre général de Walras. Les économistes et les sociologues qui s'avanceront dans ces domaines pourront utiliser le travail d'Orléan comme guide pour traiter ce qui ne l’a pas été dans l'ouvrage examiné ici.

Pour finir, il faut s'arrêter sur la conclusion de l'ouvrage qui revient sur la critique portée par le projet de «refonder l'économie ». En présentant d'une manière savoureuse le choc entre Leonard Savage et Maurice Allais à propos des choix en situation de risque, Orléan met en lumière le fait que la théorie économique mêle trois choses bien distinctes: « dire ce qui doit être, dire ce qui est, et construire le monde» (EV, p. 3 I 4). La confusion entre les deux premiers registres est bien connue et Orléan peut prendre appui sur la critique de cette confusion qu'avaient faite Durkheim et Simiand (EV, p. 316-317). Orléan se prononce sans ambigüité en faveur de l'objectif scientifique de dire et interpréter ce qui est au lieu que de s'embarquer dans l'opération de performation, pour reprendre le terme consacré, dans laquelle le «ce qui doit être» est validé non par l’observation 
des faits, mais par leur fabrication (EV, p. 325). Cela soulève un problème philosophique profond: la vérité économique est-elle un phénomène intrinsèque, hors de porté des économistes, ou bien résulte-t-elle d'une fabrication par ceux-ci des conditions institutionnelles d'émergence de résultats conformes au «ce qui doit être» selon leurs prédictions sur les conséquences du comportement intéressé des acteurs du marché? Cette dernière option est dangereuse, dit Orléan, car la «fabrication du vrai économique» laisse la porte ouverte aux intérêts marchands, extérieurs à la recherche scientifique mais désireux que cette dernière fabrique les «vérités» utiles à leurs affaires (EV, p. 323). Sans parler des conflits d'intérêt et des errements des économistes dans le domaine de la banque et de la finance, si bien mis en évidence dans les entretiens filmés d'Inside Jobs et dénoncés dans l'ouvrage de Laurent Mauduit (2012), on ne peut rester insensible devant ce risque, notamment dans une période où l'on a vu les grandes entreprises de télécommunication américaines engager la fine fleur de l'ingénierie économique des dispositifs marchands lors de la vente des droits sur les ondes hertziennes en 19949. Au-delà du débat méthodologique, il convient de noter que les économistes se sont déjà largement engagés dans la voie de la performation, dont le caractère entrepreneurial est validé par le système universitaire américain ${ }^{10}$ et sollicité par les entreprises et les agences gouvernementales. Ils sont déjà plusieurs (Leonid Hurwicz, Eric Maskin et Roger Myerson en 2007) a y avoir recueilli la récompense suprême - le prix dit Nobel d'économie. Il est difficile d'arrêter un tel mouvement. En outre, au-delà des gratifications symboliques et monétaires, cette orientation de l'économie politique est probablement une des plus «excitantes» qui soit dans le monde académique contemporain, précisément pour son caractère démiurgique - «fabriquer le social et la politique », et au niveau planétaire de surcroît lorsqu'il s'agit de l'ingénierie financière ou des marchés des droits à polluer - auquel des économistes dont la formation initiale est celle d'ingénieur ou de mathématicien appliqué ont peu de raison de vouloir résister. Orléan offre un argument plus solide lorsqu'il avance l'idée selon laquelle cette stratégie est impossible. Les relations sociales sur lesquelles reposent les échanges économiques selon l'analyse proposée dans L'Empire de la valeur sont bien plus complexes que les actions instrumentales de l'acteur économique rationnel, même lorsque ce dernier est pris dans le cadre

9 L'affaire est décrite dans l'article de John Mcmillan (1994). Elle fait aussi l'objet de commentaires de la part d'Alvin Roth (2010), l'un des économistes recrutés pour l'occasion. Les principaux économistes actifs dans cette opération ont créé l'entreprise Market Design Inc. qui propose ses services à ceux qui ont besoin de créer des dispositifs de marchés - conception d'enchères, de marchés d'appariement - selon les enjeux politiques et la nature des biens mis en marchés.

10 Dans son étude de la profession d'économistes, Marion Fourcade (2009) montre qu'aux États-Unis la discipline valorise une position académique associée à une implication dans les affaires, alors qu'en France la discipline s'est construite à l'ombre de l'État, sur la base d'une différence entre les professeurs universitaires et les économistes administratifs, donnant lieu à une intersection «limitée» avec le monde des affaires (p.225 et 247). Cette dernière caractéristique est en passe de disparaître. 
d'interactions demandant la mise en œuvre d'une rationalité stratégique et non plus seulement paramétrique. Les relations socio-économiques complexes qui forment et transforment les préférences et les identités, qui parviennent à sélectionner un objet support de confiance et de foi sociale par le mécanisme de «l'élection mimétique» ou qui définissent la convention financière à l'image du concours de beauté keynésien sont des forces «que l'économiste ne saurait fabriquer, ni même contrôler, car elles échappent radicalement à l'intentionnalité individuelle» (EV, p. 326-327). L'argument est fort car il découle directement de toute la théorie exposée dans l'ouvrage; c'est un argument empirique, au sens où il peut faire l'objet d'une falsification dès lors que tel ou tel processus relationnel est modélisé ou, au moins, fait l'objet d'une simulation assez avancée pour le transformer en un mécanisme social d'échange ou un mécanisme d’appariement. Je suis personnellement frappé par le fait que des formes de ce que j'appelle, faute de mieux, des «performations axiologiques» sont déjà à l'œuvre pour les échanges de donneurs dans la greffe rénale pour paires de donneurs-receveurs médicalement incompatibles (Steiner, 2012). Dans ce cas, certes plus limité que ceux envisagés dans l'ouvrage, ce ne sont pas les comportements instrumentaux seulement qui sont pris en charge par l'ingénierie économique d'Alvin Roth", ce sont des relations fondées sur le don, le don de vie, et cela dans le but explicite d'éviter la mise en place de marchés d'organes à transplanter. Les techniques de simulation axiologique doivent donc être suivies de près pour apprécier la portée du «théorème d'impossibilité» que propose Orléan.

\section{Références}

AGLIETTA Michel, ORLEAN André, 2002, La Monnaie entre violence et confiance, Paris, Odile Jacob.

BECKERT Jens, ASPERS Patrick, 20II, The Worth of Goods. Valuation and Pricing in the Economy, Oxford, Oxford university Press.

COCHOY Franck, DUBUISSON-QUELLIER Sophie, 2000, «Les professionnels du marché», numéro spécial de Sociologie du travail.

DURKHEIM Émile, 1970 (I886), «Les études de sciences sociales», in La Science sociale et l'action, Paris, PUF.

FOURCADE Marion, 2009, Economists and Societies. Disciplines and Profession in the United States, Britain, \& France, 1890s to 1990s, Princeton, Princeton university Press.

GODECHOT Olivier, 201I, «Finance and the rise in inequalities in France», Document de travail, Paris School of Economics.

II Rappelons qu'après des études de mathématique appliquée, ce dernier est professeur d'économie à l'université de Harvard, spécialiste de théorie des jeux, d'économie expérimentale et d'ingénierie économique. Il est également un des membres fondateurs de Market Design Inc. 
GOSSEN Hermann, 1995 (I854), Exposition des lois de l'échange et des règles de l'industrie qui s'en déduisent, trad. française, Paris, Économica.

HIRSCHMAN Albert, 1977, Les Passions et les intérêts. Justifications politiques du capitalisme avant son apogée, trad. française, Paris, PUF.

KARPIK Lucien, 2007, L'Économie des singularités, Paris, Gallimard.

MCMILLAN John, 1994, «Selling Spectrum Rights », Journal of Economic Perspectives, 8-3, p. $145-162$.

MAUDUIT Laurent, 2012, Les Imposteurs de l'économie. Comment ils s'enrichissent et nous trompent!, Paris, J.-C. Gawsewitch éditeur.

ORLEAN André, 1999, Le Pouvoir de la finance, Paris, Odile Jacob.

-, 2002, «Le tournant cognitif en économie», Revue d'économie politique, II2-5, p. $717-738$.

ROTH Alvin, 2010, «Experiments in Market design », in J. Kagel \& A. Roth, Handbook of Experimental Economics, vol. 2., à paraître.

STEINER Philippe, 2011, «The Creator, human conduct and the maximisation of utility in Gossen's economic theory», European Journal of the History of Economic Thought, 18-3, p. 353-379.

-, 2012, «Les performations économiques et axiologiques des échanges», conférence à l'Universidade Federal de Rio Grande do Sul, Gemass Working Paper, disponible sur le site du Gemass: http://www.gemass.fr

-, TRESPEUCH Marie, 2013, «La construction sociale de l'intérêt: les jeux d'argent en ligne et les organes à transplanter», Revue française de sociologie, à paraître.

\section{HÉTÉROGÉNÉITÉ DES MARCHÉS ET PRIX SANS SUBSTANCE Un commentaire de L'Empire de la valeur d'André Orléan (Seuil, 20II)}

par Richard Arena

GREDEG \& MSH, CNRS/Université de Nice-Sophia Antipolis arena.unice@gmail.com

L’ouvrage d'André Orléan - L’Empire de la valeur - présente deux caractéristiques. D’un côté, la publication de ce livre intervient dans une période où, selon l'auteur, «l'économie en tant que discipline traverse [...] une grave crise de légitimité» (EV, p. 9). La crise financière de 2008 aurait ainsi permis de mettre en lumière les erreurs et les limites de la théorie économique moderne et, en particulier, de celles qui sont liées à l'un de ses fondements essentiels, le concept de «valeur économique». D’un autre côté, l'ouvrage d’André Orléan offre au lecteur une synthèse à la fois convaincante et innovante de beau- 